\title{
IMPACT OF THE STRESS FACTOR ON THE PRICE OF WIDOW'S PENSIONS. PROOFS
}

UDC 519.21

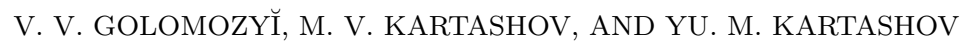

\begin{abstract}
This is a continuation of a recent paper by the authors (in Modern Problems in Insurance Mathematics, E. A. A. Series, Springer, New York, 2014, pp. 223237) containing proofs of the results announced therein. We consider the model of joint life insurance with a stress factor. The proofs use the method of a maximal coupling for time inhomogeneous Markov chains. The stability of expectations of a function of a Markov moment is proved.
\end{abstract}

\section{INTRODUCTION}

This paper contains proofs of the results announced in 11. The proofs are based on the coupling method developed in [6] and allow us to extend the results of [1] to the case of time inhomogeneous Markov chains.

The coupling method and method of maximal coupling are explained in detail by Lindvall in 8 .

In the current paper, we consider a discrete phase space, although an analogous construction of maximal coupling can be developed in the general case as well. An appropriate description of this construction for time homogeneous Markov chains can be found in [8, p. 18] (see the section in [8] devoted to $\gamma$-coupling).

We apply some general ideas concerning the stability of Markov chains and processes presented in 4,5 . Some basic results of the modern theory of the stochastic stability of Markov chains are contained in 9 .

The first author's papers [2, 3] contain some results on the stochastic stability obtained by using the coupling method for Markov chains (including the time inhomogeneous case). On the other hand, these papers do not discuss results on the functionals of the stopping times.

The paper [1] provides an approximation of premiums for the joint life insurance in the case of a small stress factor. Some numerical examples can also be found in [1.

The numbers of the theorems, definitions, lemmas, and equations below coincide with the corresponding numbers of theorems, definitions, lemmas, and equations in [1] if they start with the prefix 14 .

\section{MAin DEFinitions}

Consider two time inhomogeneous Markov chains $X$ and $X^{\prime}$ with the discrete phase space $E=\{i, j, k, l, \ldots\}$. Denote by $P_{t}=\left(P_{i j}^{(t)}\right)$ and $P_{t}^{\prime}=\left(P_{i j}^{\prime(t)}\right)$ their matrices of transient probabilities at step $t$, that is, $P_{i j}^{(t)}\left(P_{i j}^{\prime(t)}\right)$ is the probability that the chain

2010 Mathematics Subject Classification. Primary 60J45; Secondary 60A05, 60K05.

Key words and phrases. Coupling method, maximal coupling, discrete Markov chains, stability of distributions of Markov chains. 
$X\left(X^{\prime}\right)$ is in the state $j$ at the moment $t+1$ provided $X\left(X^{\prime}\right)$ was in the state $i$ at the moment $t$. The probability measures and expectations of the chains $X$ and $X^{\prime}$ that start from a state $i$ at the moment $t=0$ are denoted by $\mathrm{P}_{i}, \mathrm{E}_{i}$ and $\mathrm{P}_{i}^{\prime}, \mathrm{E}_{i}^{\prime}$, respectively.

The transient probabilities over $n \geq 1$ steps from time $t$ to time $t+n$ are given by the matrices

$$
P^{(t, n)}=\prod_{s=t}^{t+n-1} P_{s}, \quad P^{\prime(t, n)}=\prod_{s=t}^{t+n-1} P_{s}^{\prime}, \quad t \geq 0 .
$$

Below we use the following notation for the relative difference of transient probabilities and the mixing coefficient over one step for chains $X$ and $X^{\prime}$ :

$$
\rho_{t}(i, j)=\left(P_{i j}^{(t)}-P_{i j}^{(t)}\right)^{+} / P_{i j}^{(t)}, \quad \rho_{t}^{\prime}(i, j)=\left(P_{i j}^{(t)}-P_{i j}^{(t)}\right)^{+} / P_{i j}^{\prime(t)},
$$

where $x^{+}=\max (x, 0)$ and

$$
\varepsilon_{t}=\sup _{i, j \in E} \rho_{t}(i, j), \quad \varepsilon_{t}^{\prime}=\sup _{i, j \in E} \rho_{t}^{\prime}(i, j), \quad t \geq 0 .
$$

We agree that $0 / 0=0$.

Consider the $\sigma$-algebras $\mathfrak{F}_{n}=\sigma\left[X_{t}, t \leq n\right]$ and $\mathfrak{F}_{n}^{\prime}=\sigma\left[X_{t}^{\prime}, t \leq n\right]$. Assume that both values $X_{0}$ and $X_{0}^{\prime}$ are nonrandom and fixed.

Let $\theta \geq 1$ be a stopping time with respect to the flow of $\sigma$-algebras $\left(\mathfrak{F}_{n}\right)$ and let a $\mathfrak{F}_{\theta}$-measurable function $\varphi$ be constructed from a family of sets $\left\{B_{n}, n \geq 1\right\}, B_{n} \subset E^{n}$, and functions $\varphi_{n}: E^{n} \rightarrow \mathbb{R}_{+}$such that

$$
\{\theta=n\}=\left\{\left(X_{1}, \ldots, X_{n}\right) \in B_{n}\right\}, \quad \varphi \mathbb{1}_{\{\theta=n\}}=\varphi_{n}\left(X_{1}, \ldots, X_{n}\right)
$$

for all $n \geq 1$.

Definition 14.1. A pair $\left(\theta^{\prime}, \varphi^{\prime}\right)$, where $\theta^{\prime}$ is a $\left(\mathfrak{F}_{n}^{\prime}\right)$-stopping time and $\varphi^{\prime}$ is a $\mathfrak{F}_{\theta^{\prime}}^{\prime}$ measurable random variable, is said to correspond to a pair $(\theta, \varphi)$ if the random events $\left\{\theta^{\prime}=n\right\}$ and random variables $\varphi^{\prime} \mathbb{1}_{\left\{\theta^{\prime}=n\right\}}$ are constructed from $\left(X_{1}^{\prime}, \ldots, X_{n}^{\prime}\right)$ by using the same sets $B_{n}$ and functions $\varphi_{n}$ as in (3).

Theorem 14.1. Let $\theta \geq 1$ be a $\left(\mathfrak{F}_{n}\right)$-stopping time. Assume that a random variable $\varphi$ is nonnegative and $\mathfrak{F}_{\theta}$-measurable. Further let a pair $\left(\theta^{\prime}, \varphi^{\prime}\right)$ correspond to the pair $(\theta, \varphi)$. Then

$$
\mathrm{E}_{i}[\varphi \underline{\varepsilon}(\theta)]-\mathrm{E}_{i}^{\prime}\left[\varphi^{\prime} \bar{\varepsilon}^{\prime}\left(\theta^{\prime}\right)\right] \leq \mathrm{E}_{i} \varphi-\mathrm{E}_{i}^{\prime} \varphi^{\prime} \leq \mathrm{E}_{i}[\varphi \bar{\varepsilon}(\theta)]-\mathrm{E}_{i}^{\prime}\left[\varphi^{\prime} \underline{\varepsilon}^{\prime}\left(\theta^{\prime}\right)\right]
$$

if the random variables $\bar{\varepsilon}(\theta), \underline{\varepsilon}(\theta), \bar{\varepsilon}^{\prime}\left(\theta^{\prime}\right)$, and $\underline{\varepsilon}^{\prime}\left(\theta^{\prime}\right)$ are such that

$$
\begin{gathered}
\bar{\varepsilon}(\theta) \geq 1-\prod_{s=0}^{\theta-1}\left(1-\rho_{s}\left(X_{s}, X_{s+1}\right)\right) \geq \underline{\varepsilon}(\theta) \geq 0, \\
\bar{\varepsilon}^{\prime}\left(\theta^{\prime}\right) \geq 1-\prod_{s=0}^{\theta^{\prime}-1}\left(1-\rho_{s}^{\prime}\left(X_{s}^{\prime}, X_{s+1}^{\prime}\right)\right) \geq \underline{\varepsilon}^{\prime}\left(\theta^{\prime}\right) \geq 0
\end{gathered}
$$

almost surely.

Remark 1. By definition (2), inequalities (5) and (6) hold if

(7) $\bar{\varepsilon}(\theta)=1-\prod_{t=0}^{\theta-1}\left(1-\varepsilon_{t}\right), \quad \underline{\varepsilon}(\theta)=0, \quad \bar{\varepsilon}^{\prime}\left(\theta^{\prime}\right)=1-\prod_{t=0}^{\theta^{\prime}-1}\left(1-\varepsilon_{t}^{\prime}\right), \quad \underline{\varepsilon}^{\prime}\left(\theta^{\prime}\right)=0$.

Example 1. Let two stopping times $\theta$ and $\theta^{\prime}$ be such that

$$
\{\theta=n\}=\left\{\left(X_{1}, \ldots, X_{n}\right) \in B_{n}\right\}, \quad\left\{\theta^{\prime}=n\right\}=\left\{\left(X_{1}^{\prime}, \ldots, X_{n}^{\prime}\right) \in B_{n}\right\}
$$

for all $n \geq 1$ and $B_{n} \subset E^{n}$. 
Then

$$
\begin{gathered}
-\sum_{t \geq 0} \varepsilon_{t}^{\prime} \mathrm{P}_{i}^{\prime}\left(\theta^{\prime} \in T, \theta^{\prime}>t\right) \leq \mathrm{P}_{i}(\theta \in T)-\mathrm{P}_{i}^{\prime}\left(\theta^{\prime} \in T\right) \leq \sum_{t \geq 0} \varepsilon_{t} \mathrm{P}_{i}(\theta \in T, \theta>t), \\
-\sum_{t \geq 0} \varepsilon_{t}^{\prime} \mathrm{E}_{i}^{\prime}\left(\theta^{\prime} \mathbb{1}_{\theta^{\prime}>t}\right) \leq \mathrm{E}_{i} \theta-\mathrm{E}_{i}^{\prime} \theta^{\prime} \leq \sum_{t \geq 0} \varepsilon_{t} \mathrm{E}_{i}\left(\theta \mathbb{1}_{\theta>t}\right)
\end{gathered}
$$

for all $T \subset \mathbb{Z}_{+}$. Indeed, choose $\varphi=\mathbb{1}_{\theta \in T}$. Then the condition in Definition 14.1 follows from (8). Using (2) and (17) we obtain the bound

$$
\mathrm{E}_{i}\left[\mathbb{1}_{\theta \in T} \bar{\varepsilon}(\theta)\right] \leq \mathrm{E}_{i}\left[\mathbb{1}_{\theta \in T} \sum_{t=0}^{\theta-1} \varepsilon_{t}\right]=\sum_{t \geq 0} \mathrm{P}_{i}[\theta \in T, \theta>t] \varepsilon_{t},
$$

whence the second inequality in (4) follows.

An analogous inequality holds for $\theta^{\prime}$ as well. Thus property (9) is proved.

The proof of property (10) for $\varphi=\theta$ is similar and uses the inequality

$$
\mathrm{E}_{i}[\varphi \bar{\varepsilon}(\theta)] \leq \mathrm{E}_{i}\left[\theta \sum_{t=0}^{\theta-1} \varepsilon_{t}\right]=\sum_{t \geq 0} \mathrm{E}_{i}\left[\theta \mathbb{1}_{\theta>t}\right] \varepsilon_{t} .
$$

\section{Proof OF RESUlts}

Consider the chain $\bar{X}=\left(X_{t}, X_{t}^{\prime}, d_{t}, t \geq 0\right)$ defined in sections 14.3.2-14.3.4 of the paper [1], where

$$
d_{t}=\mathbb{1}_{\left\{X_{t}=X_{t}^{\prime}\right\}}, \quad t \geq 0 .
$$

Denote by $\tau$ the first decoupling moment for the chain $\bar{X}$ that starts from the state $(i, i, 1)$ :

$$
\tau=\inf \left\{t \geq 1: d_{t}=0\right\} .
$$

Lemma 14.1. Let $i_{0}=i \in E$ be an arbitrary initial state. Let $\left\{i_{1}, i_{2}, \ldots, i_{s+1}\right\} \subset E$, $0 \leq s<t$, be an arbitrary family of states. Then

$$
\begin{gathered}
\mathrm{P}_{i i 1}\left[\tau=s+1 \mid \tau>s, X_{1}=i_{1}, \ldots, X_{t}=i_{t}\right]=\rho_{s}\left(i_{s}, i_{s+1}\right), \\
\mathrm{P}_{i i 1}\left[\tau>s \mid X_{1}=i_{1}, \ldots, X_{t}=i_{t}\right]=\prod_{r=0}^{s-1}\left(1-\rho_{r}\left(i_{r}, i_{r+1}\right)\right), \\
\mathrm{P}_{i i 1}\left[\tau=s+1 \mid X_{1}=i_{1}, \ldots, X_{t}=i_{t}\right]=\rho_{s}\left(i_{s}, i_{s+1}\right) \prod_{r=0}^{s-1}\left(1-\rho_{r}\left(i_{r}, i_{r+1}\right)\right) .
\end{gathered}
$$

Proof. Following the paper [1] we introduce the notation

$$
\begin{gathered}
Q_{i j}^{(s)}=\min \left(P_{i j}^{(s)}, P_{i j}^{(s)}\right)=\mathrm{P}_{i i 1}\left(\tau>s, X_{s}=j\right)=P_{i j}^{(s)}-R_{i j}^{(s)}, \\
R_{i j}^{(s)}=\left(P_{i j}^{(s)}-P_{i j}^{(s)}\right)^{+}=\rho_{s}(i, j) P_{i j}^{(s)} .
\end{gathered}
$$

Given $i_{1}, \ldots, i_{t} \in E$ and $0 \leq s<t$ we introduce the random events

$$
\begin{gathered}
A_{s}=\left\{\bar{X}_{1}=\left(i_{1}, i_{1}, 1\right), \ldots, \bar{X}_{s}=\left(i_{s}, i_{s}, 1\right)\right\}, \\
C_{s+1}=\left\{X_{s+1}=i_{s+1}, \ldots, X_{t}=i_{t}\right\} .
\end{gathered}
$$


The inequalities $X_{r}=X_{r}^{\prime}, r \leq s$, hold if and only if the random event $\{\tau>s\}$ occurs. Thus the left hand side of (12) is equal to

$$
\begin{aligned}
\mathrm{P}_{i i 1}[\tau= & \left.s+1 \mid A_{s}, C_{s+1}\right]=\sum_{l \in E} \mathrm{P}_{i i 1}\left[\bar{X}_{s+1}=\left(i_{s+1}, l, 0\right) \mid A_{s}, C_{s+1}\right] \\
= & \sum_{l \in E} \mathrm{P}_{i i 1}\left[\bar{X}_{s+1}=\left(i_{s+1}, l, 0\right), A_{s}, C_{s+1}\right] / \mathrm{P}_{i i 1}\left[A_{s}, C_{s+1}\right] \\
= & \sum_{l \in E} \mathrm{P}_{i i 1}\left[\bar{X}_{s+1}=\left(i_{s+1}, l, 0\right), C_{s+1} \mid A_{s}\right] / \mathrm{P}_{i i 1}\left[C_{s+1} \mid A_{s}\right] \\
= & \sum_{l \in E} \mathrm{P}_{i i 1}\left[\bar{X}_{s+1}=\left(i_{s+1}, l, 0\right), C_{s+1} \mid \bar{X}_{s}=\left(i_{s}, i_{s}, 1\right)\right] / \mathrm{P}_{i i 1}\left[C_{s+1} \mid \bar{X}_{s}=\left(i_{s}, i_{s}, 1\right)\right] \\
= & \sum_{l \in E} \mathrm{P}_{i i 1}\left[\bar{X}_{s+1}=\left(i_{s+1}, l, 0\right) \mid \bar{X}_{s}=\left(i_{s}, i_{s}, 1\right)\right] \\
& \times \mathrm{P}_{i i 1}\left[C_{s+1} \mid \bar{X}_{s}=\left(i_{s}, i_{s}, 1\right), \bar{X}_{s+1}=\left(i_{s+1}, l, 0\right)\right] / \mathrm{P}_{i i 1}\left[C_{s+1} \mid \bar{X}_{s}=\left(i_{s}, i_{s}, 1\right)\right] \\
= & \sum_{l \in E} h_{i_{s}, i_{s+1} l}^{(s)} \prod_{r=s+1}^{t} P_{i_{r}, i_{r+1}}^{(r)} / \prod_{r=s}^{t} P_{i_{r}, i_{r+1}}^{(r)}=\sum_{l \in E} h_{i_{s}, i_{s+1} l}^{(s)} / P_{i_{s}, i_{s+1}}^{(s)} \\
= & R_{i_{s}, i_{s+1}}^{(s)} / P_{i_{s}, i_{s+1}}^{(s)}=\rho_{s}\left(i_{s}, i_{s+1}\right) .
\end{aligned}
$$

Here we used the Markov property of the chain $\bar{X}$, relations (14.13) and (14.25) in the paper [1, and the following equalities:

$$
\begin{aligned}
\sum_{l \in E} h_{i, j l}^{(s)} / P_{i j}^{(s)} & =\sum_{l \in E} R_{i j}^{(s)} R_{i l}^{(s)} /\left(1-q_{i}^{(s)}\right) P_{i j}^{(s)}=R_{i j}^{(s)} / P_{i j}^{(s)}=\left(P_{i j}^{(s)}-Q_{i j}^{(s)}\right) / P_{i j}^{(s)} \\
& =\rho_{s}(i, j) .
\end{aligned}
$$

This proves equality (12). Further, the left hand side of (13) is equal to

$$
\begin{aligned}
\mathrm{P}_{i i 1}[ & \left.A_{s} \mid X_{1}=i_{1}, \ldots, X_{t}=i_{t}\right] \\
& =\mathrm{P}_{i i 1}\left[A_{s}, X_{1}=i_{1}, \ldots, X_{t}=i_{t}\right] / \mathrm{P}_{i i 1}\left[X_{1}=i_{1}, \ldots, X_{t}=i_{t}\right] \\
& =\mathrm{P}_{i i 1}\left[A_{s}, C_{s+1}\right] / \mathrm{P}_{i i 1}\left[X_{1}=i_{1}, \ldots, X_{t}=i_{t}\right] \\
& =\mathrm{P}_{i i 1}\left[A_{s}\right] \mathrm{P}_{i i 1}\left[C_{s+1} \mid A_{s}\right] / \mathrm{P}_{i i 1}\left[X_{1}=i_{1}, \ldots, X_{t}=i_{t}\right] \\
& =\left(\prod_{r=0}^{s-1} Q_{i_{r}, i_{r+1}}^{(r)}\right)\left(\prod_{r=s}^{t-1} P_{i_{r}, i_{r+1}}^{(r)}\right) /\left(\prod_{r=0}^{t-1} P_{i_{r}, i_{r+1}}^{(r)}\right) \\
& =\prod_{r=0}^{s-1} Q_{i_{r}, i_{r+1}}^{(r)} / P_{i_{r}, i_{r+1}}^{(r)}=\prod_{r=0}^{s-1}\left(1-R_{i_{r}, i_{r+1}}^{(r)} / P_{i_{r}, i_{r+1}}^{(r)}\right)=\prod_{r=0}^{s-1}\left(1-\rho^{(r)}\left(i_{r}, i_{r+1}\right)\right) .
\end{aligned}
$$

An expression for the probability $\mathrm{P}_{i i 1}\left[A_{s}\right]$ used above follows from the Markov property of the chain $\bar{X}$. We also used the equality

$$
\mathrm{P}\left[\tau>s+1, \bar{X}_{s+1}=(j, j, 1) \mid \tau>s, \bar{X}_{s}=(i, i, 1)\right]=Q_{i j}^{(s)}
$$

that follows from (14.13).

Finally, equality (14) is obtained by multiplying together relations (12) and (13).

Proof of Theorem 14.2. Note that $\left(X_{1}, \ldots, X_{\theta}\right)=\left(X_{1}^{\prime}, \ldots, X_{\theta}^{\prime}\right)$ in the random event $\{\theta<\tau\}$. Hence $\varphi=\varphi^{\prime}$ for this event and, by the definition of the corresponding 
pair,

$$
\begin{aligned}
\mathrm{E}_{i i 1}[\varphi, \theta<\tau] & =\sum_{t \geq 1} \mathrm{E}_{i i 1}\left[\varphi \mathbb{1}_{\{\theta=t\}}, t<\tau\right] \\
& =\sum_{t \geq 1} \mathrm{E}_{i i 1}\left[\varphi_{t}\left(X_{1}, \ldots, X_{t}\right) \mathbb{1}_{\left\{\left(X_{1}, \ldots, X_{t}\right) \in B_{t}\right\}} \mathbb{1}_{\{\theta=t\}}, t<\tau\right] \\
& =\sum_{t \geq 1} \mathrm{E}_{i i 1}\left[\varphi_{t}^{\prime}\left(X_{1}^{\prime}, \ldots, X_{t}^{\prime}\right) \mathbb{1}_{\left\{\left(X_{1}^{\prime}, \ldots, X_{t}^{\prime}\right) \in B_{t}\right\}} \mathbb{1}_{\left\{\theta^{\prime}=t\right\}}, t<\tau\right] \\
& =\mathrm{E}_{i i 1}\left[\varphi^{\prime}, \theta^{\prime}<\tau\right] .
\end{aligned}
$$

Then

$$
\mathrm{E}_{i} \varphi-\mathrm{E}_{i}^{\prime} \varphi^{\prime}=\mathrm{E}_{i i 1}[\varphi, \theta \geq \tau]-\mathrm{E}_{i i 1}\left[\varphi^{\prime}, \theta^{\prime} \geq \tau\right] .
$$

Now we use (3), (13), and (14.4) and apply Lemma 4.1 to obtain

$$
\begin{aligned}
\mathrm{E}_{i i 1}[\varphi, \theta \geq \tau] & =\sum_{t \geq 1} \mathrm{E}_{i i 1}\left[\varphi \mathbb{1}_{\{\theta=t\}}, \tau \leq t\right] \\
& =\sum_{t \geq 1} \mathrm{E}_{i i 1}\left[\mathbb{E}\left(\varphi_{t}\left(X_{1}, \ldots, X_{t}\right) \mathbb{1}_{\{\theta=t\}} \mathbb{1}_{\{\tau \leq t\}} \mid X_{1}, \ldots, X_{t}\right)\right] \\
& =\sum_{t \geq 1} \mathrm{E}_{i i 1}\left[\varphi_{t}\left(X_{1}, \ldots, X_{t}\right) \mathbb{1}_{\{\theta=t\}} \mathbb{P}\left(\tau \leq t \mid X_{1}, \ldots, X_{t}\right)\right] \\
& =\sum_{t \geq 1} \mathrm{E}_{i i 1}\left[\varphi_{t}\left(X_{1}, \ldots, X_{t}\right) \mathbb{1}_{\{\theta=t\}}\left(1-\prod_{s=0}^{t-1}\left(1-\rho_{s}\left(X_{s}, X_{s+1}\right)\right)\right)\right] \\
& =\sum_{t \geq 1} \mathrm{E}_{i i 1}\left[\varphi_{t}\left(X_{1}, \ldots, X_{t}\right) \mathbb{1}_{\{\theta=t\}}\left(1-\prod_{s=0}^{\theta-1}\left(1-\rho_{s}\left(X_{s}, X_{s+1}\right)\right)\right)\right] \\
& \leq \sum_{t \geq 1} \mathrm{E}_{i i 1}\left[\varphi_{t}\left(X_{1}, \ldots, X_{t}\right) \mathbb{1}_{\{\theta=t\}} \bar{\varepsilon}(\theta)\right]=\sum_{t \geq 1} \mathrm{E}_{i i 1}\left[\varphi \mathbb{1}_{\{\theta=t\}} \bar{\varepsilon}(\theta)\right] \\
& =\mathrm{E}_{i i 1}[\varphi \bar{\varepsilon}(\theta)]=\mathrm{E}_{i}[\varphi \bar{\varepsilon}(\theta)] .
\end{aligned}
$$

Here we used relation (14.25).

Combining (16) and the latter inequalities we get bound (44). The inequality mentioned in Remark 1 follows from (2).

The expression in the third line from bottom in inequality (16) is estimated by $\underline{\varepsilon}(\theta)$ defined in (5). Hence

$$
\begin{aligned}
& \mathrm{E}_{i i 1}[\varphi, \theta \geq \tau] \leq \sum_{t \geq 1} \mathrm{E}_{i i 1}\left[\varphi_{t}\left(X_{1}, \ldots, X_{t}\right) \mathbb{1}_{\{\theta=t\}} \bar{\varepsilon}(\theta)\right]=\mathrm{E}_{i}[\varphi \bar{\varepsilon}(\theta)], \\
& \mathrm{E}_{i i 1}[\varphi, \theta \geq \tau] \geq \sum_{t \geq 1} \mathrm{E}_{i i 1}\left[\varphi_{t}\left(X_{1}, \ldots, X_{t}\right) \mathbb{1}_{\{\theta=t\}} \underline{\varepsilon}(\theta)\right]=\mathrm{E}_{i}[\varphi \underline{\varepsilon}(\theta)] .
\end{aligned}
$$

A bound for $\mathrm{E}_{i i 1}\left[\varphi^{\prime}, \theta^{\prime} \geq \tau\right]$ is proved similarly.

Substituting these four inequalities into (15), we obtain two inequalities in (4).

\section{BIBLIOGRAPHY}

1. Y. Kartashov, V. Golomozyı̆, and N. Kartashov, The impact of stress factors on the price of widow's pensions, Modern Problems in Insurance Mathematics (D. Silvestrov and A. MartinLöf, eds.), E. A. A. Series, Springer, 2014, pp. 223-237. MR3330687

2. V. V. Golomozyŭ, Stability of time in-homogeneous Markov chains, Visn. Kyiv. Univer. Ser. Phys. Math. 4 (2009), 10-15. (Ukrainian) 
3. V. V. Golomozy $\breve{1}$, A subgeometrical estimate for stability for time-homogeneous Markov chains, Teor. Ímovir. Mat. Stat. 81 (2009), 35-50; English transl. in Theor. Probability and Math. Statist. 81 (2010), 31-46. MR2667308

4. N. V. Kartashov, Strong Stable Markov Chains, VSP/"TViMS", Utrecht/Kiev, The Netherlands/Ukraine, 1996. MR 1451375

5. N. V. Kartashov, The ergodicity and stability of quasi-homogeneous Markov semigroups of operators, Teor. Ǐmovir. Mat. Stat. 72 (2005), 54-62; English transl. in Theor. Probability and Math. Statist. 72 (2006), 59-68.

6. N. V. Kartashov and V. V. Golomozyı̆, Maximal coupling procedure and stability of discrete Markov chains. I, Teor. Ǐmovir. Mat. Stat. 86 (2012), 81-92; English transl. in Theor. Probability and Math. Statist. 86 (2013), 93-104. MR.2986452

7. N. V. Kartashov and V. V. Golomozyŭ, Maximal coupling procedure and stability of discrete Markov chains. II, Teor. Imovir. Mat. Stat. 87 (2012), 47-59; English transl. in Theor. Probability and Math. Statist. 87 (2013), 65-78. MR3241447

8. T. Lindvall, Lectures on Coupling Method, Dover Publication, 2002. MR.1924231

9. S. P. Meyn and R. L. Tweedie, Markov Chains and Stochastic Stability, Springer-Verlag, 1993. MR 1287609

Department of Probability Theory, Statistics, and Actuarial Mathematics, Faculty for Mechanics and Mathematics, Taras Shevchenko National University of Kyiv, Academician Glushkov Avenue, 6, Kyiv 03127, Ukraine

Department of Probability Theory, Statistics, and Actuarial Mathematics, Faculty for Mechanics and Mathematics, Taras Shevchenko National University of Kyiv, Academician Glushkov Avenue, 6, Kyiv 03127, Ukraine

E-mail address: nkartashov@skif.com.ua

Department of Probability Theory, Statistics, and Actuarial Mathematics, Faculty for Mechanics and Mathematics, Taras Shevchenko National University of Kyiv, Academician Glushkov Avenue, 6, Kyiv 03127, Ukraine

Received 23/DEC/2014

Translated by S. KVASKO 